Herz 2012 · 37:585-588

DOI 10.1007/s00059-012-3657-2

Online publiziert: 8 . August 2012

(c) Urban \& Vogel 2012

S. Pankuweit · J. Schäfer · M. Schoppet

Klinik für Kardiologie, Universitätsklinikum Gießen und Marburg GmbH, Standort Marburg, Marburg

\title{
Zur Emeritierung von Prof. Dr. Bernhard Maisch im September 2012
}

\section{Prof. Dr. Matthias Rothmund, Dekan des Fachbereichs Medizin der Philipps- Universität Marburg:}

Als Kollege und Nachfolger von Prof. Dr. Bernhard Maisch im Amt des Dekans ist es für mich ein Anliegen, ihm für seine erfolgreiche Arbeit im Fachbereich der Philipps-Universität zu danken.

Nachdem sich Prof. Maisch auf die Aufgaben eines Lehrstuhlinhabers und Klinikdirektors in Tübingen und später in Würzburg vorbereitet hatte, übernahm er den Lehrstuhl für Kardiologie in Marburg im Jahre 1989. Mit der für ihn typischen Energie und Schaffenskraft machte er die Klinik in wenigen Jahren zu einer Anlaufstelle für Herzkranke aus der Stadt, der Region und vor allem auch für Patienten aus der gesamten Bundesrepublik.

Seiner Energie ist die Gründung einer herzchirurgischen Klinik im Jahre 1994 mitzuverdanken. Diese Klinik war für ihn Voraussetzung zur Akquisition von Patienten mit schwierigen Krankheitsbildern seines Fachs und für Interventionen, die im Laufe der Jahre immer anspruchsvoller wurden. Schwerpunkt der Klinik für Kardiologie unter seiner Leitung waren zweifellos die Kardiomyopathien, die chronische Herzinsuffizienz und kardiologische Erkrankungen bei Patienten mit angeborenen Stoffwechselstörungen.

Prof. Maisch war in den Jahren von 2002 bis 2007 Dekan des Fachbereichs Medizin. In diesen Zeitraum fielen die Fusion und Privatisierung der Universitätskliniken in Gießen und Marburg und die Entstehung des Universitätskli- nikums Gießen und Marburg (UKGM) $\mathrm{GmbH}$.

Der Dekan Maisch zeichnete sich in dieser für den Fachbereich und das Klinikum schwierigen Zeit als entschiedener Gegner einer Privatisierung aus und schlug der Politik immer wieder alternative Modelle in unterschiedlicher Trägerschaft vor, musste aber schließlich an der Beratungsresistenz der Meinungsbildner und Entscheider in der Landesregierung scheitern. Die Zeitläufte bis in das Frühjahr des Jahres 2012 gaben ihm im Nachhinein recht, nachdem man das in der Bundesrepublik bislang einmalige Experiment der Privatisierung eines hochschulmedizinischen Standorts als gescheitert bezeichnen musste.

Der Kampf gegen die Privatisierung sowie die ersten Jahre der Kooperation

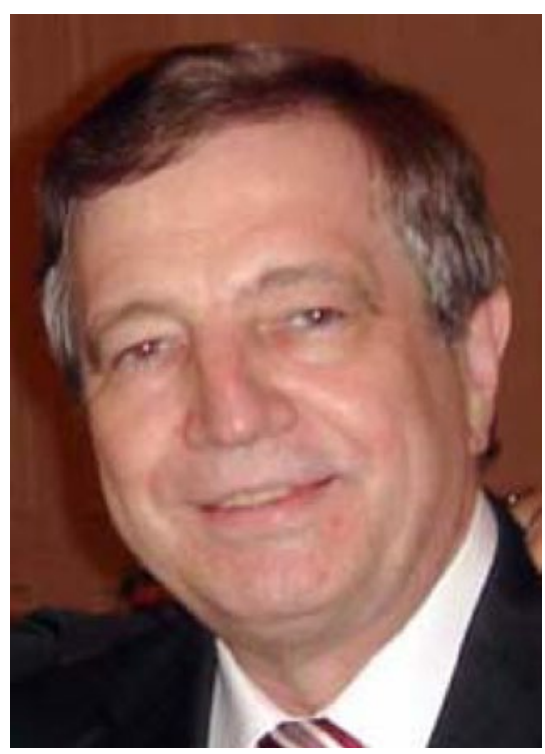

Abb. 1 A Prof. Dr. Bernhard Maisch, 2007 
mit dem privaten Klinikumsbetreiber haben ihn viel Kraft gekostet. Alle, die seine Arbeit beobachteten, waren erstaunt über den kaum zu übertreffenden Arbeitseinsatz in dieser Zeit. Man erhielt von ihm E-Mails in seiner Funktion als Klinikdirektor und Dekan in der tiefen Nacht und am frühen Morgen, zusätzlich zu dem Arbeitspensum, was er über Tag zwischen 6.00 Uhr morgens und 22.00 Uhr abends absolvierte.

Seine Zeit als Dekan war auch gekennzeichnet von dem Versuch, eine Transparenz und Konsolidierung der Finanzen des Fachbereichs zu erreichen. Dies war nötig geworden, nachdem das Land Hessen entschieden hatte, seinen Zuführungsbetrag nicht mehr der Medizin direkt, sondern der Universität zuzuweisen. Mit der ihm eigenen Hartnäckigkeit hat Dekan Maisch gegen viele Widerstände erhebliche Fortschritte, wenn auch nicht das erstrebte Ziel, in dieser Frage erreicht.

Prof. Dr. Bernhard Maisch hat in 23 Jahren seiner Tätigkeit die Geschicke der Klinik für Kardiologie mit sicherer Hand in zukunftsfähige Bahnen geleitet und vor allem als Dekan dem Fachbereich in schwierigsten Zeiten unverzichtbare Dienste geleistet.

Der Dank seiner Kollegen und seines Nachfolgers begleitet ihn in die nächste Phase seines Lebens.

\section{Prof. Dr. Georg Ertl, Direktor der Medizinischen Klinik und Poliklinik I am Universitäts- klinikum Würzburg, Präsident der Deutschen Gesellschaft für Kardiologie - Herz- und Kreislaufforschung, Sprecher des Kompetenznetzes Herzinsuffizienz:}

Gerne möchte ich hier im Namen der Deutschen Gesellschaft für Kardiologie - Herz- und Kreislaufforschung, des Kompetenznetzes Herzinsuffizienz und als Kollege aus der Würzburger Zeit die Leistungen von Herrn Prof. Bernhard Maisch würdigen. Wir kennen uns, seit sich unsere Wege Anfang der 80er-Jahre in Würzburg gekreuzt haben - zunächst beide als wissenschaftliche Assistenten, später als Oberärzte.
Bernhard Maisch brachte das für ihn in seinem beruflichen Leben zentrale Thema der Immunpathogenese von Herzkrankheiten schon aus Tübingen mit nach Würzburg. Er hatte seine Doktorarbeit im Physiologischen Institut bei Ruthard Jacob verfertigt und war dann wissenschaftlicher Assistent an der Medizinischen Universitätsklinik bei Kurt Kochsiek geworden. Nach einem Forschungsaufenthalt 1975 im Department für Immunologie am Middlesex Hospital bei Ivan Roitt schloss er sich, nach Tübingen zurückgekehrt, in den Jahren von 1975 bis 1980 in seiner Forschung eng an Peter A. Berg an, dem er seine Ausbildung in der Immunologie verdankt. 1978 unterbrach er seine klinische Ausbildung nochmals für einen Forschungsaufenthalt am INSERM Paris, im Institut für Biochemie bei Bernard Swynghedauw.

Bernhard Maisch begleitete 1980 Kurt Kochsiek nach Würzburg, wo er jung Oberarzt und Professor und später leitender Oberarzt an der Medizinischen Universitätsklinik Würzburg wurde. Neben der kreativ und mit großem Engagement weiter vorangetrieben kardioimmunologischen Forschung, die ihn auch auf das weitere Gebiet der entzündlichen Herzkrankheiten führte, ließ sich Bernhard Maisch in Würzburg immer wieder von seinen klinischen Aufgaben zu neuen Forschungsthemen inspirieren. So nutzte er beispielsweise seine Verantwortung für die Schrittmachertherapie für hämodynamische Untersuchungen zur physiologischen Schrittmacherstimulation oder die Einführung der transösophagealen Echokardiographie für Untersuchungen zu entzündlichen Herzkrankheiten. Bernhard Maisch ist auf dem Gebiet der Kardiomyopathien und insbesondere ihrer Immunpathogenese ein weltweit anerkanntes und geschätztes Mitglied der internationalen Forschergemeinde. 1989 nahm Professor Maisch den Ruf auf eine C4-Professur an der Philipps-Universität Marburg an, wo er zunächst die Einrichtung der Herzchirurgie erkämpfte und dann zusammen mit dem Herzchirurgen Rainer Moosdorf ein Herzzentrum einrichtete.

Bernhard Maisch ist seit 1979 Mitglied der Deutschen Gesellschaft für Kardiologie - Herz- und Kreislaufforschung
(DGK). Über die Jahre war er Mitglied der Arbeitsgruppen „Vaskuläre Biologie“, „Kardiovaskulärer Ultraschall", „Interventionelle Kardiologie“, „Chronische Herzinsuffizienz“, „Kardiomyopathien“, „Thorakale Organtransplantation" und „Magnetresonanzverfahren in der Kardiologie“. Er ist German Fellow der Europäischen Gesellschaft für Kardiologie (ESC) und Gutachter der ESC und DGK. Als gewählter Tagungspräsident für die Herbsttagung der DGK 2004 war er von 2003 bis 2004 Mitglied der für unsere Gesellschaft so wichtigen Programmkommission, die für das wissenschaftliche Programm der Tagungen der Gesellschaft verantwortlich zeichnet. Er wurde dann zusammen mit Prof. Pfeiffer Tagungspräsident bei der sehr erfolgreichen 28 . Herbsttagung und 15. Jahrestagung der Arbeitsgruppe Herzschrittmacher und Arrhythmie vom 14. bis 16. Oktober 2004 in Hamburg. Vielbeachtetes Tagungsthema war das Management kardiovaskulärer Erkrankungen. 2004 war er auch Mitglied im Kuratorium für den Hans-Jürgen-Bretschneider-Posterpreis. Schließlich diente Prof. Maisch von 2010 bis 2012 als Mitglied der Kommission für Wahlvorschläge für die DGK. Über viele Jahre war Prof. Maisch gefragter Dozent im Dienste der DGK.

Prof. Maisch hat, unterstützt durch seine Mitarbeiter, insbesondere Frau PD Dr. Sabine Pankuweit, schon bei der Gründung des Kompetenznetzes Herzinsuffizienz (KNHI) mitgewirkt, das seit Juni 2003 durch das Bundesministerium für Bildung und Forschung gefördert wird. Die Marburger Arbeitsgruppe hat sich den wichtigen Zielen des KNHI, die unter dem Motto „Mehrwert durch Vernetzung" stehen, verschrieben und die leistungsstarke Infrastruktur für Kooperationsvorhaben, insbesondere klinische Studien und Register, mit aufgebaut. So wurden in den acht Jahren der bisherigen Förderung rund 10.000 herzinsuffiziente Patienten in die Teilprojekte sowie weitere etwa 4.000 Patienten in assoziierte Projekte von Studien des KNHI aufgenommen und ein Großteil über längere Zeiträume (3-6 Jahre) nachbeobachtet. Für alle Patienten in diesem großen Kollektiv stehen nun ein gleicher Basisdatensatz und je eine Blutprobe in der Bioma- 
terialbank für weitere Forschung zur Verfügung. Das Potenzial dieses durch die Standardisierung im Netz so wertvollen Daten- und Materialschatzes ist hoch, die Auswertungen stehen erst am Anfang. Prof. Bernhard Maisch hat als Vorstandsmitglied und stellvertretender Sprecher des KNHI wesentlich zu diesen Erfolgen beigetragen.

Die Deutsche Gesellschaft für Kardiologie - Herz- und Kreislaufforschung und das Kompetenznetz Herzinsuffizienz danken Bernhard Maisch für seine Leistungen für die Kardiologie und in der Herzkreislaufforschung. Seine Freunde freuen sich auf einen auch in Zukunft kreativen Geist und aktiven Mitstreiter für die Herz-Kreislauf-Forschung.

\section{Dr. Perry Elliott, University College London, Centre for Cardiology in the Young, Reader in Inherited Cardiovascular Disease, Chairman of the WG 21"Myocardial and Pericardial Diseases" of the European Society of Cardiology:}

As current chairman of the European Society of Cardiology Working Group for Myocardial and Pericardial Diseases, it gives me enormous pleasure to make this tribute to Professor Bernhard Maisch on the occasion of his retirement as Chairman of the Department of Internal Medicine and Cardiology of the University Hospital of the Philipps-University Marburg.

For almost three decades, Professor Maisch has combined an illustrious career as an experienced and wise physician with that of an active researcher in the field of heart muscle disease. His major area of interest has been inflammatory and autoimmune mechanisms in myocardial and pericardial disease and over the course of his productive career he has written more than 300 papers, making significant contributions to our understanding of the pathogenesis, clinical diagnosis and treatment of myocarditis and pericarditis. He has influenced national and international policy making and scientific endeavour through his chairmanship of major international bodies including the International Society and
Federation of Cardiology and the European Society of Cardiology (ESC) working group for myocardial and pericardial diseases. Through his stewardship of major international meetings and workshops in the University of Marburg, he has been a leading educator and inspiration for the scientists and clinicians of the future. More recently, Bernhard has provided me with wise counsel and advice and has been a major contributor to the pericardial and myocarditis task forces of our working group.

Retirement of an eminent member of our community is always a bitter sweet event; on the one hand we lose an eminent contributor to the life blood of the cardiological community; on the other, we have an opportunity to honour a steadfast friend and colleague. On behalf of all the members of the working group and the wider community of doctors and scientists active in the field of heart muscle disease, I thank Bernhard for his great contribution over the years and wish him and his family good fortune in the future.

\section{Mitarbeiter der Klinik für Kardiologie des Uni- versitätsklinikums Gießen und Marburg GmbH:}

Nach der Emeritierung von Prof. Dr. Gustav Adolf Martini als Lehrstuhlinhaber der Inneren Medizin (1963-1981) am Universitätsklinikum Marburg erfolgte in den 90er-Jahren eine umfassende und weitreichende Umstrukturierung in der Inneren Medizin mit Bildung eigenständiger Abteilungen. Im Rahmen dieser Neustrukturierung wurde 1984 Prof. Dr. Bodo Strauer als erster Ordinarius auf den neu eingerichteten Lehrstuhl für Kardiologie berufen. Nach dessen Wechsel an die Universität Düsseldorf im Jahre 1987 folgte 1989 der damals 42-jährige Bernhard Maisch als zweiter Lehrstuhlinhaber der Kardiologie in Marburg - ein Lehrstuhl, den er über 23 Jahre innehatte (- Abb. 2).

Nach dem Studium in Tübingen, Lausanne und Hamburg sowie der Approbation im Jahre 1974 folgten Assistentenjahre an der Universitätsklinik Tübingen unter Prof. Dr. Kochsiek. Nach dem Wechsel von Prof. Kochsiek an die Uni-

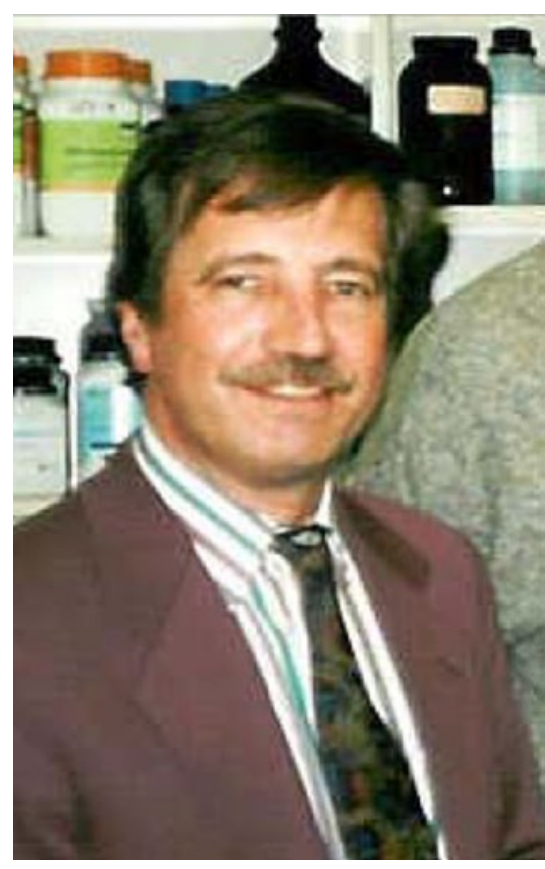

Abb. 2 А Prof. Dr. Bernhard Maisch, 1996

versitätsklinik Würzburg folgte ihm Prof. Maisch dorthin, wurde Oberarzt und zuletzt leitender Oberarzt. Bereits in Tübingen wurde der Grundstein für das wissenschaftliche Interesse von Prof. Maisch auf dem Gebiet der Kardiomyopathien gelegt. Nach Promotion im Jahre 1975 folgte die Habilitation 1984 zum Thema „Untersuchungen zu humoralen und zellulären Reaktionen des Immunsystems bei kardialen Erkrankungen“. Nach dem Wechsel von Würzburg nach Marburg wurde dieses Thema weiterverfolgt, und Prof. Maisch war in verschiedenen nationalen und internationalen Arbeitsgruppen zu diesem Themenkreis tätig. So war er 1982 Mitbegründer der Arbeitsgruppe „Entzündliche Herzmuskelerkrankungen“ der Deutschen Gesellschaft für Kardiologie, 1987 Mitbegründer der Working Group 21 - Myocardial and Pericardial Diseases der European Society of Cardiology (ESC), Mitglied des Council of Cardiomyopathies der ISFC/World Heart Federation von 1986 bis 2004 und ist Fellow der European Society of Cardiology sowie des American College of Cardiology. Neben der Leitung der Abteilung für Innere Medizin und Kardiologie als Klinikdirektor folgte in den Jahren von 2002 bis 2007 die Tätigkeit als - damals noch „nebenamtlicher“ - Dekan der Medizinischen Fakultät der Philipps-Universität. 
Die ursprünglich auf drei Jahre angelegte Amtszeit wurde auf Wunsch des Fachbereichsrats um eine zweite Amtsperiode verlängert, da die Neuwahl durch die politischen Wirren mit Fusion und Privatisierung in eine der schwierigsten Zeiten der 480-jährigen Geschichte der Marburger Universitätsmedizin gefallen wäre. Trotz der enormen Arbeitsbelastung stimmte Prof. Maisch einer zweijährigen Verlängerung seiner Amtszeit als Dekan zu. Während dieser Zeit kämpfte er für die Freiheit der Forschung und hatte weitreichende Vorbehalte gegen eine privatisierte Trägerschaft einer unabhängigen Universitätsmedizin. Zur damaligen Zeit wurde der Fachbereich Medizin von der Kernuniversität mehr oder weniger alleingelassen. Seine Hartnäckigkeit, sein Mut und sein Verhandlungsgeschick, gerade auch in den Zeiten der Privatisierung des Universitätsklinikums, wurden bewundert und anerkannt. Betrachtet man die jetzigen Debatten um die $\mathrm{Zu}$ kunft des Universitätsklinikums Gießen und Marburg (UKGM) unter privater Trägerschaft, sollte er recht behalten, und man hat das Gefühl, dass er hier fast hellseherische Fähigkeiten hatte.

In der langen Zeit des Ordinariats wurde die Marburger Kardiologie zu einem Kompetenzzentrum auf dem Gebiet der inflammatorischen Kardiomyopathie mit nationaler und internationaler Reputation. Darüber hinaus gelangen die Einwerbung von zwei Stiftungsprofessuren sowie die Einbindung in nationale Forschungsverbünde wie z. B. das BMBF-geförderte Kompetenznetz Herzinsuffizienz. Als Hochschullehrer hielt Prof. Maisch die Hauptvorlesung stets selbst und gestaltete darüber hinaus auch den Kleingruppenunterricht sowie Seminare und Visiten für die Studenten, die dies mit Anerkennung und hervorragenden Evaluationen honorierten. Die Arbeitsatmosphäre in der von ihm geführten Klinik war trotz zahlreicher Probleme, die bei angespannter Personaldecke und hoher Arbeitsbelastung auf allen Ebenen oftmals unausweichlich sind, dennoch familiär und vertrauensvoll. Was Prof. Maisch nach langen Jahren harter Arbeit in Zukunft machen wird, hat er noch nicht verraten, sicher aber wird er der Medizin nicht komplett den Rücken kehren. Wir wünschen ihm einen erfüllenden „Unruhestand“ als Emeritus und die Gelassenheit, die im hektischen klinischen Alltag manchmal fehlt. Alle Mitarbeiterinnen und Mitarbeiter der Klinik für Kardiologie wünschen Prof. Dr. B. Maisch alles Gute für eine hoffentlich interessante und gesunde Zukunft und freuen sich mit ihm gemeinsam auf das 7th International Symposium on Inflammatory Heart Diseases am 21. und 22. September 2012 in Marburg.

\section{S. Pankuweit, J. Schäfer, M. Schoppet, im Juni 2012}

\section{Korrespondenzadresse}

\section{PD Dr. S. Pankuweit}

Klinik für Kardiologie, Universitätsklinikum

Gießen und Marburg GmbH,

Standort Marburg

Baldingerstraße, 35033 Marburg

pankuwei@staff.uni-marburg.de 